\title{
Compact Ka-Band Lens Antennas for LEO Satellites
}

\author{
Jorge R. Costa, Member, IEEE, Carlos A. Fernandes, Member, IEEE, Gaël Godi, \\ Ronan Sauleau, Senior Member, IEEE, Laurent Le Coq, and Hervé Legay
}

\begin{abstract}
Two new compact lens antenna configurations are presented and compared for data link communications with LEO satellites at $26 \mathrm{GHz}$. These lenses match a secant type radiation pattern template in the elevation plane while having a mechanically scanned sector beam in azimuth to enhance gain as much as possible. No rotary joints or multiple feeds are required and emphasis is put also on the compactness of the proposed solutions $\left(<6 \lambda_{0}\right)$. Two alternative lens configurations are evaluated numerically and experimentally: one is based on modified axial-symmetric dome lens geometry, and the other one consists of a full 3-D double-shell lens antenna. In contrast to current nearly omnidirectional antennas, the directivity of our lens prototypes is above $15.4 \mathrm{dBi}$. Up to $4.2 \mathrm{~dB}$ loss obtained in the prototypes can be significantly reduced by using lower loss dielectrics and matching layers, without affecting the conclusions. The numerical and experimental results are in good agreement with the radiation specifications given the compact size of the antennas.
\end{abstract}

Index Terms-Beam shaping, compact lens antennas, constant flux illumination, scanning antennas.

\section{INTRODUCTION}

D ATA LINK antennas used in Low Earth Orbit (LEO) satellites for global Earth observation offer a constant flux coverage of the Earth surface. Ground stations are typically viewed from the satellite within a cone of $65^{\circ}$ half angle. Due to the Earth curvature, the path between the ground station and the satellite, and consequently the associated attenuation, increase as the elevation angle is moving closer to $\pm 65^{\circ}$. The antenna EIRP shall therefore compensate this loss attenuation dependence with $\theta$, leading to a typical template with a wide illumination angle $\left( \pm 65^{\circ}\right)$, a deep inlet in the center region and a fast roll off outside the coverage area.

Manuscript received October 18, 2007; revised December 19, 2007. This work was supported in part by the ACE Network of Excellence (activity 2.1) and in part by the CNRS and the Conseil Régional de Bretagne, France (MINICANT and CREATE/CONFOCAL projects).

J. R. Costa is with the Instituto de Telecomunicações, IST, 1049-001 Lisboa, Portugal and also with the Instituto Superior de Ciências do Trabalho e da Empresa, Departamento de Ciências e Tecnologias da Informação, 1649-026 Lisboa, Portugal (e-mail: Jorge.Costa@1x.it.pt).

C. A. Fernandes is with Instituto de Telecomunicações, IST, 1049-001 Lisboa, Portugal.

Gaël Godi was with the Institut d'Electronique et de Télécommunications de Rennes (IETR), UMR CNRS 6164, Université de Rennes 1, 35042 Rennes, France-AUTHOR: SINCE RECEIVING THE Ph.D. DEGREE, WHAT ORGANIZATION IS GODI WITH NOW? (e-mail: Ronan.Sauleau@univrennes1.fr)

R. Sauleau and L. Le Coq are with Institut d'Electronique et de Télécommunications de Rennes (IETR), UMR CNRS 6164, Université de Rennes 1, 35042 Rennes, France (e-mail: Ronan.Sauleau@univ-rennes1.fr).

H. Legay is with Thales Alenia Space, 31037 Toulouse, France (e-mail: herve. legay@thalesaleniaspace.com).

Digital Object Identifier 10.1109/TAP.2008.922690
There are a number of antenna solutions for covering this need, ranging from electronically and mechanically steerable antennas to passive isoflux antennas. Steerable antennas offer high EIRP beams which allows relaxing the power to be supplied by the amplifier modules. The main drawback of active antennas is their complexity and cost [1], and that of mechanically steerable antennas is the single point failure of its 2 axes mechanism scheme, which also requires a rotary joint. On the contrary, isoflux antennas can not exceed typically $4 \mathrm{dBi}$ at the End of Antenna Coverage, and a high power amplifier shall be used. Existing antennas are based on reflector assemblies, typically with fixed omnidirectional beam, so with low directivity [2]-[4], or combined with multiple feeds for sector coverage [5]. These structures are bulky, with diameter and height larger than $10 \lambda_{0}$. Known solutions based on dome lenses target wide angle pencil-beam scanning rather than sec shaped beams and these structures are also larger than $10 \lambda_{0}$ [6], [7] and are typically combined with complex feeding arrays.

Data Transmission Systems are currently implemented in the highest majority in X band (typically between 8025 and 8350 $\mathrm{MHz}$ ). In order to avoid the congestion of this band, new data transmission systems are being defined in Ka-band, where a band is reserved for such applications. As lens antennas are good candidates at millimeter waves [8]-[10], the present paper intends to explore and compare alternative lens configurations candidate for such missions. The main challenge is to find lens solutions that offer the best tradeoff between the following simultaneous requirements:

a) Shaped sec type elevation beam with the highest possible directivity;

b) Very compact antenna solution (diameter $<6 \lambda_{0}$ and height $<4 \lambda_{0}$ ), to cope with mass and volume restrictions;

c) Compatibility with mechanical azimuth scanning of the shaped beam without needing multiple feeds or any rotary joints, ensuring uncomplicated assembly and reliable operation.

The feasibility of designing small shaped lenses with pencil beams has been investigated numerically in a prior work [11]. In contrast to [11], and prior lens works [12] which focused on validation of the numerical tools, the emphasis of this paper is clearly on the development and assessment of a new lens antenna concept that excels previous antenna solutions for this application. The very small lens size $\left(\sim 6 \lambda_{0}\right.$ in diameter $)$ has implications on gain template compliance [11], [13] since a good match usually requires at least a $20 \lambda_{0}$ diameter lens [13]. One of the major objectives of the current paper is thus to evaluate how close a sec type of radiation pattern can be approached with the new small lightweight lens configurations. Indicative target specifications are given in Table I. 
TABLE I

LENS TARGET SPECIFICATIONS

\begin{tabular}{|c|l|l|}
\hline$\#$ & Frequency & $26 \mathrm{GHz}$ \\
\hline 1 & $\mathrm{~S}_{11}$ on $50 \Omega$ & $\leq-10 \mathrm{~dB}$ \\
\hline 2 & Polarization & $\begin{array}{l}\text { RHCP }(\text { Axial ratio }<7 \mathrm{~dB}) \\
\text { Or LP }\end{array}$ \\
\hline 3 & Elevation aperture & $0-65^{\circ}$ off-nadir \\
\hline 4 & $\begin{array}{l}\text { Gain template in } \\
\text { elevation plane }\end{array}$ & $\begin{array}{c}\text { Max at } 65^{\circ} \\
>-10.5 \mathrm{~dB} \text { at } 40^{\circ} \\
<-13 \mathrm{~dB} \text { at } 0^{\circ}\end{array}$ \\
\hline 5 & Azimuth template & $\begin{array}{l}\text { The one providing the best } \\
\text { possible gain }(>5.5 \mathrm{dBi})\end{array}$ \\
\hline 6 & Weight & $<200 \mathrm{~g}$ \\
\hline
\end{tabular}

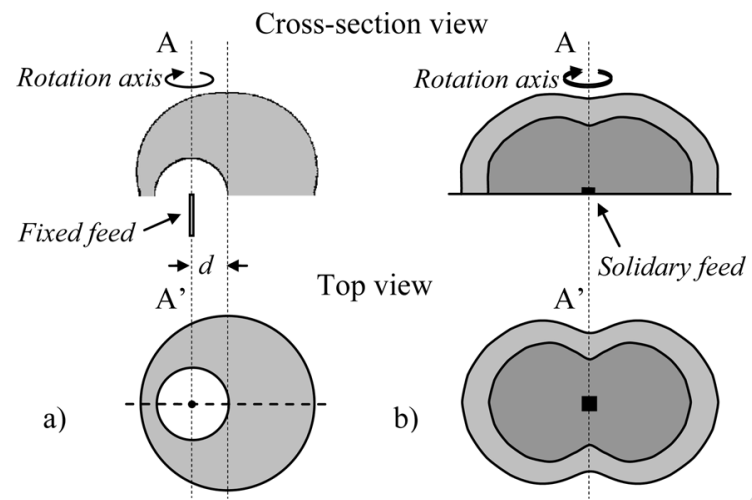

Fig. 1. General geometry of the proposed antennas: (a) modified axial-symmetric shaped dome lens (L1); (b) full 3-D lens (L2).

This paper is organized as follows. The lens concept is presented in Section II. The dome lens antenna and the 3-D lens design and performance are presented in Sections III and IV respectively. In both cases, numerical and experimental results are given and discussed. Conclusions are finally drawn in Section V.

\section{LENS CONCEPT}

The proposed new antenna configurations consist either of a modified axial-symmetric shaped dome lens [see Fig. 1(a)] or of a full 3-D lens formed by two shells of different dielectric materials [Fig. 1(b)]. Throughout this work, these lenses are referred to as L1 and L2, respectively.

In L1 lens design, the inner shell is actually a spherical air cavity that is displaced radially together with the feed by distance $d$ with respect to the axis of the otherwise axial-symmetric outer shell. The asymmetry introduced by the feed position pro duces an adequate directive shaped beam as shown in Section II. This is a very simple and light solution where the feed is fixed and the lens rotates about the AA' axis to provide the necessary beam scanning. The feed is not in physical contact with the lens, thus avoiding the need for fault prone rotary joints in long continuous usage.

In the L2 lens, a double-material (double-shell) configuration is used. This ensures a better control of the azimuth beamwidth favoring an increased gain and improved power transmission across the lens interfaces [14]. In this case, the feed is centered with the lens. Although it is fixed at the lens base in the presented study, an air cavity can also be used to allow mechanical revolving of the lens about the AA' axis for azimuth beam scan

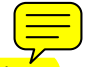

without needing a solidary-AUTHOR: SHOULD THIS BE "solitary"? feed.

In this paper the lens feed is based on printed antenna technology; however it is noteworthy to mention that the lens configuration can be easily adjusted to other feeds like waveguides. Either circularly-polarized (CP) feeds or linearly-polarized (LP) feeds are used ahead, depending on the lens configuration.

The lens shape is calculated from geometrical optics (GO) [13] and, in the 3-D case, it is further optimized using genetic algorithms (GA) coupled with geometrical optics/physical optics (GO/PO) method of analysis [12], [15]. Two antenna prototypes were fabricated and experimentally tested providing satisfactory results.

Macor and Rexolite materials are used for the lens prototypes. Although these materials are not space-qualified and the first one presents moderately high losses, they have been selected to prove the concepts. Re-design of the presented lens configurations for other dielectric materials is straightforward.

\section{OfF-AXIS Fed AXIAL-Symmetric DOME LENS}

The modified axial-symmetric dome lens (L1) is designed to produce a sector beam with a secant type elevation pattern. The proposed concept is the following: the lens combined with a $\mathrm{CP}$ feed allows mechanical scanning of the beam by rotating (by $360^{\circ}$ ) just the lens above the feed, while maintaining the feed at a fixed position. A $10 \mathrm{~mm}$ radius spherical air cavity at the lens base, concentric with the feed phase center, avoids contact between the feed and the lens while it rotates. This concept was pre-evaluated only by simulation in [16], using a LP feed.

The main characteristics of the required $\mathrm{CP}$ feed and of the developed lens are given in Section III-A. Experimental results are discussed in Section III-B.

\section{A. Lens Design}

The lens feed is a diagonally probe-fed rectangular patch antenna with $3.22 \mathrm{~mm} \times 3.48 \mathrm{~mm}$ dimensions manufactured using a 10 mils-thick Rogers Duroid 5880 substrate. The patch produces a right-hand circular polarization (RHCP) far-field radiation pattern (in-air)

An axial symmetrical lens can provide the required shaped sector beam solution by appropriately displacing the feed away from the lens axis [17]. The design of such a lens involves three steps, as explained hereafter.

In the first step, an axial symmetric homogeneous lens with n-axis feed (centered with AA' axis) is designed using GO formulation [see Fig. 2(a)]. A closed-form analytical expression can be derived for the lens surface profile complying with $\sec (k \theta)$ output power elevation function [9], which contains the very loose gain template from Table I. The dielectric material used for this lens is Macor $\left(\varepsilon_{\mathrm{r}}=5.5 \times(1-\mathrm{j} 0.0118)\right)$. Since this is a completely axial symmetrical lens, the horizontal plane ray tracing is uniform [see Fig. 2(b)].

In the second step, a new lens is generated by rotating the bold part of the original profile around a displaced vertical axis BB' [see Fig. 2(c)]. Although a smaller lens is obtained, the feed is maintained in its original position from step 1, resulting in off-axis feeding position $(-d, 0,0)$. Because the bold part of profile and the feed distance are preserved, in the vertical plane 

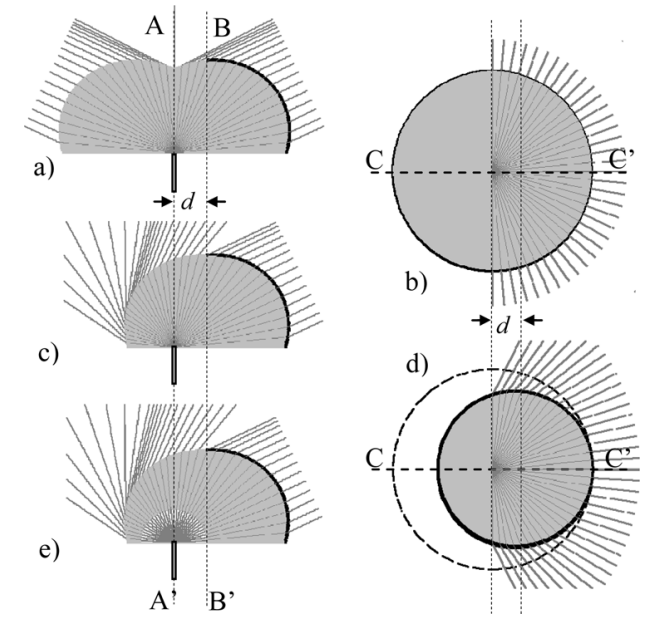

Fig. 2. L1 lens design steps. (a), (c), and (e) correspond to len along CC'. (b) Top view of the (a) lens. (d) Top view of the (2).

containing the $\mathrm{CC}^{\prime}$ axis, the ray tracing near the bold part of the profile is exactly the same as in the lens from step 1 . This means that the elevation radiation pattern is approximately maintained in this plane. But now, in the horizontal plane, the off-axis position of the feed causes a refraction effect that narrows the beam in this plane [Fig. 2(d)]. This increases the antenna gain when compared to the lens at step 1 . The $d$ parameter is optimized to obtain a compromise between gain enhancement and ghily of the shaped elevation pattern within the sectored beam a spherical portion of the Macor material centered with the feed phase center is removed from the step 2 lens [Fig. 2(e)]. Because the air cavity is centered with the feed, no refraction occurs at the new inner air/Macor interface and so the radiation pattern of this lens (L1) is almost the same as for step 2. It was verified that reflection at this interface does not influence significantly the required lens shape. The air cavity allows for contactless rotation of the lens around the fixed feed.

After the GO lens design, a PO analysis was performed to obtain the actual lens performance, taking into account diffraction effects and internal reflections, which are left out by GO formulation. Our own dedicated lens design and optimization tool-ILASH-[18], based on the classical (GO/PO) combination, is used for this purpose where GO is implemented for evaluating the field distribution over the outer lens surface, and PO is used to calculate its contribution to the far-field radiation pattern. Calculated and measured results show that, for the final lens (step 3), the desired elevation pattern remains reasonably constant within a prescribed azimuth interval, depending on the above referred axis displacement $d$. Optimization of the design led to $d=9.7 \mathrm{~mm}$. The final lens is $50 \mathrm{~mm}$ in diameter that is, $<5 \lambda_{0}$ and $30 \mathrm{~mm}$ height $\left(<3 \lambda_{0}\right)$.

The simulated lens radiation patterns in both principal planes are represented in Fig. 3. As expected, the radiation pattern cannot be as sharp as the $\sec (k \theta)$ function in the plane $\phi=0^{\circ}$, given the reduced lens dimensions. Although the calculated pattern exceeds the analytical template used for the lens design, it matches the loose amplitude template from Table I. The excess of the calculated curve over the analytical template could be anticipated from the stray rays exiting the lens near $\theta=30^{\circ}$
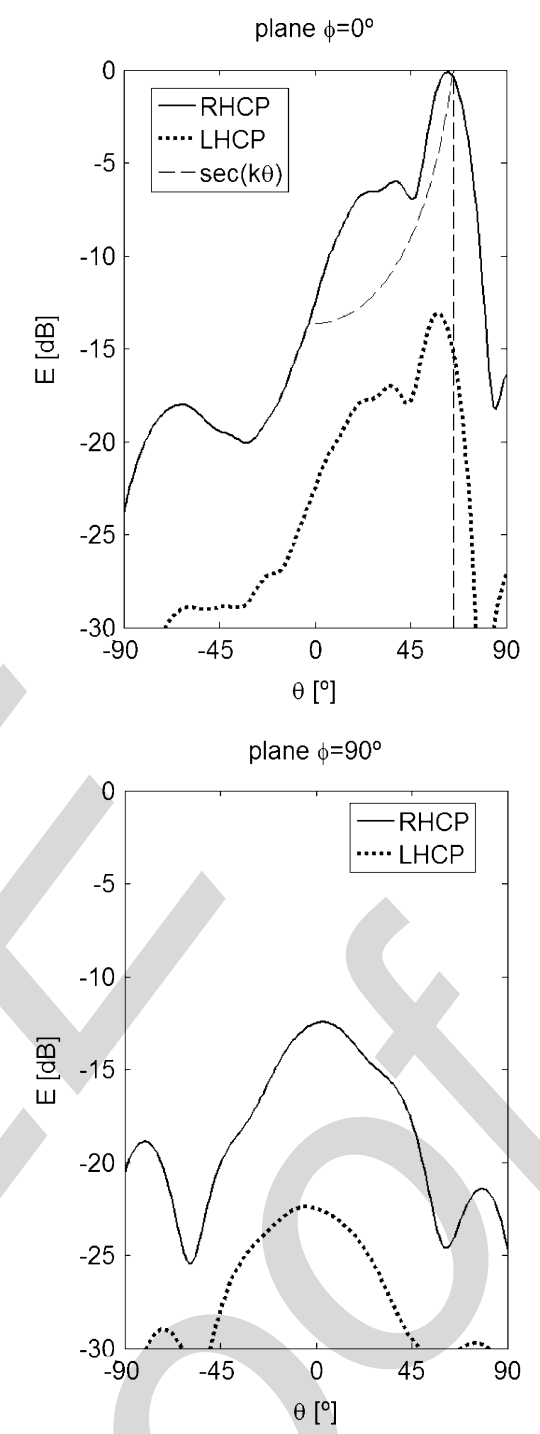

Fig. 3. Simulated sector beam $\mathrm{L} 1$ lens radiation pattern computed with $\mathrm{GO} / \mathrm{PO}$ at $26 \mathrm{GHz}$.

in Fig. 2(e). Finally, it is noted that radiation in the $\phi=90^{\circ}$ plane is clearly lower than for $\phi=0^{\circ}$ plane, confirming that the axial-symmetric L1 lens with off-axis feed can in fact produce a radiation pattern with sector characteristic.

This property is clearly highlighted in Fig. 4 which shows in 3-D the simulated far-field co- and cross-polarization components of the lens when using simulated $\mathrm{CP}$ feed radiation patterns. The maximum directivity is $15.4 \mathrm{dBi}$ at $\theta=61^{\circ}$. However, due to Macor high dissipation loss and to internal reflection losses at the two interfaces (numerically estimated to be of the order of $2.2 \mathrm{~dB}$ and $2 \mathrm{~dB}$, respectively), lens gain is estimated to be around $11.2 \mathrm{dBi}$. The maximum level of the cross-polarization component is $-13.1 \mathrm{~dB}$. The axial ratio value of the lens around the maximum radiation sector is about $3 \mathrm{~dB}$ which, in large part, is due to the poor axial ratio of the feed. Further simulations using a perfect $\mathrm{CP}$ feed have confirmed that the degradation of the lens radiation pattern axial ratio amounts to a maximum of $2 \mathrm{~dB}$. The same conclusion was obtained experimentally when testing this lens with a linearly polarized printed feed. 


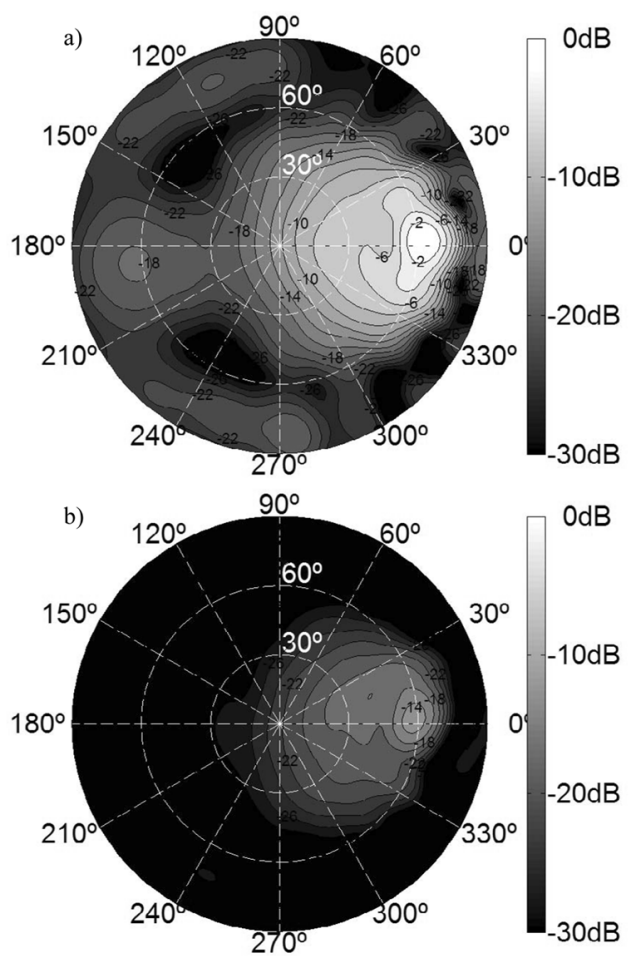

Fig. 4. Radiation performance (in polar coordinates) of the L1 lens computed with the GO/PO method. (a) RHCP component; (b) LHCP component.

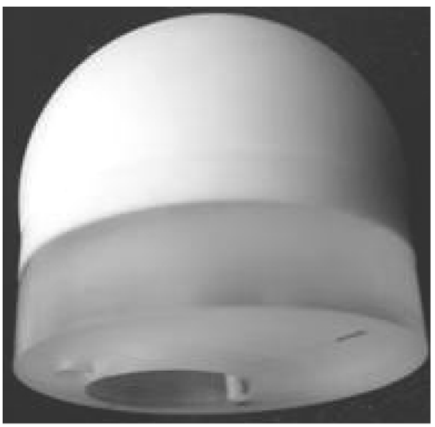

a)

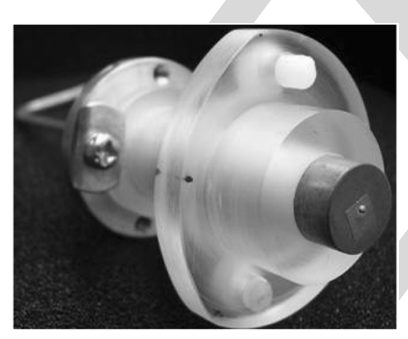

b)

Fig. 5. (a) Macor axial symmetric $50 \mathrm{~mm}$ diameter shaped lens (L1), with an off-axis air dome; (b) CP probe-fed patch feed with a small ground plane.

\section{B. Measurements}

The lens prototype has been fabricated [Fig. 5(a)] using a 3 -axis milling machine. An Acrylic base was glued at the bottom just for fixing the lens to the antenna support. The weight of the lens is only $103 \mathrm{~g}$, excluding the feed and its mechanical supports [Fig. 5(b)].

The measured input return loss of the patch is represented in Fig. 6, both for free space radiation and when positioned at the lens focus at the center of the air cavity. Lens reflection, especially at the spherical air/Macor interface, produces a shift of the frequency response. This effect can be reduced by using a matching layer at this interface, but this was not found necessary at this point to prove the lens concept.

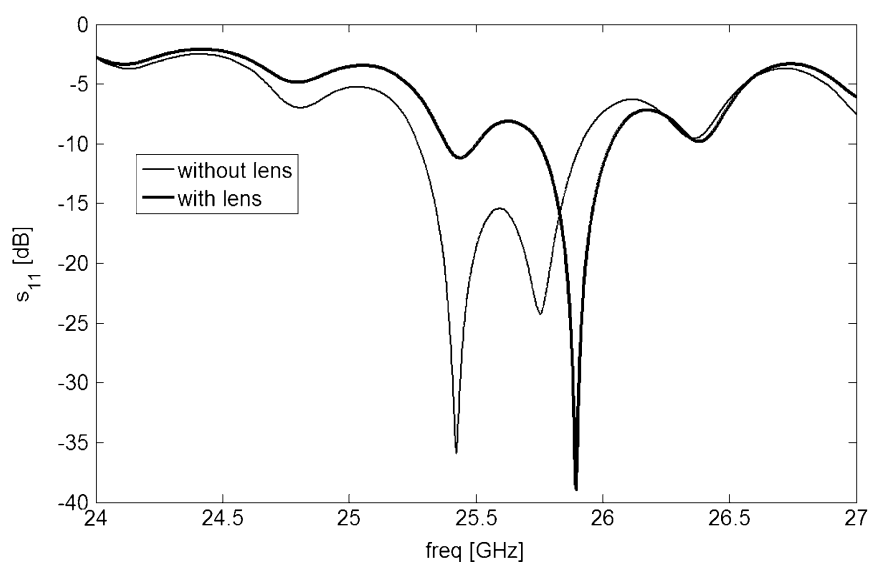

Fig. 6. Measured reflection coefficient of the patch antenna and the L1 lens antenna.
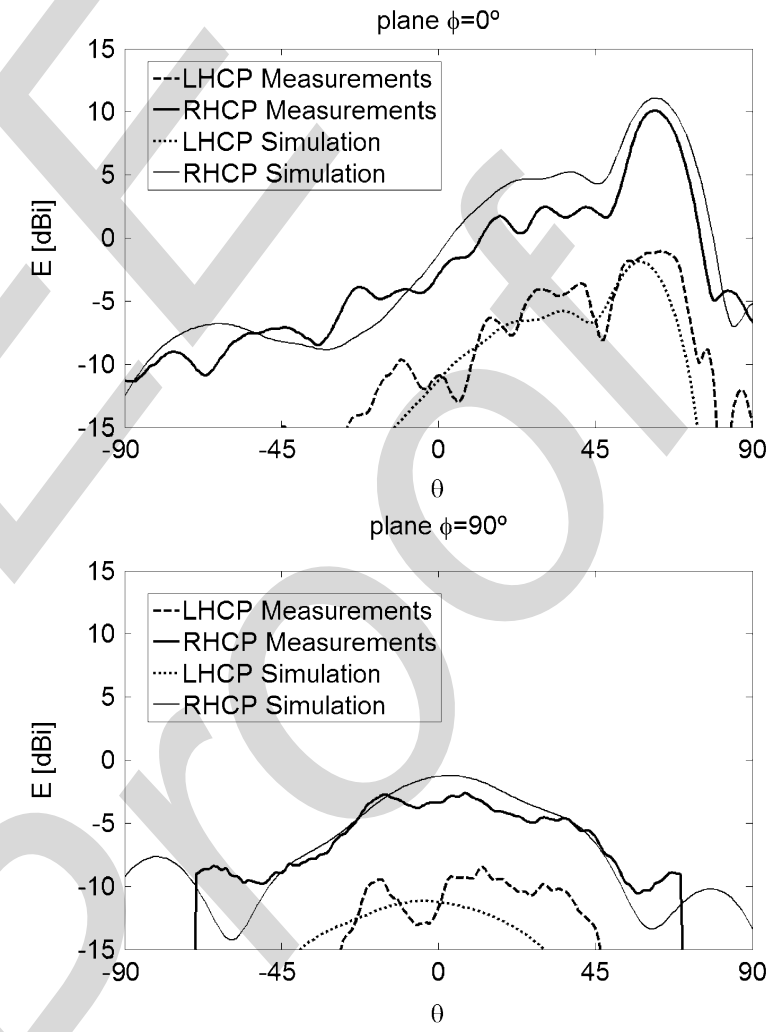

Fig. 7. Measured radiation patterns of the $\mathrm{L} 1$ lens at $26 \mathrm{GHz}$.

The experimental radiation patterns of the lens are given in Fig. 7 The measured gain is $10.4 \mathrm{dBi}$, which is reasonably close to ILASH simulation value (11.2 $\mathrm{dBi})$.

It can be seen in Fig. 7 that, around the direction of maximum radiation $\left(\theta=61^{\circ}\right)$, the measured cross-polarization is -11.3 $\mathrm{dB}$. As discussed before, this high value can be easily reduced by improving the polarization purity of the $\mathrm{CP}$ wave radiated by the feed. Nevertheless, this result is still in agreement with the specifications given in Table I.

In summary, the proposed compact $<5 \lambda_{0}$ diameter axial symmetrical shaped lens with off-axis feed can produce the desired shaped sector beam complying with target specifications, 
while being very lightweight. It is stressed that the obtained directivity is $15.4 \mathrm{dBi}$ and, if required, the $4.2 \mathrm{~dB}$ loss, can be significantly reduced by using a lower loss material and matching layers: simulations show that in this case losses reduce down to about $1 \mathrm{~dB}$ for loss tangent in the order of 0.001 .

\section{3-D DOUBLE-SHELL LENS}

A 3-D double-shell lens antenna (L2) has been also designed for a $180^{\circ}$ mechanically-scanned sector beam coverage pattern. This geometry enables one, simultaneously, to synthesize the $\sec (k \theta)$ type power template in the elevation plane, and to enhance the gain. The inner and outer shells are made of Macor and Rexolite $\left(\varepsilon_{\mathrm{r}}=2.53 \times(1-\mathrm{j} 0.0025)\right)$, respectively. As previously referred, the choice of these material permittivities allows good power transfer efficiency through the lens while maintaining a compact size. Single-shell geometries have also been tested, using either Rexolite or Macor dielectrics. In the first case (Rexolite lens), the radiation performance complies with the specifications, but the lens is too large $(150 \mathrm{~mm}$ in diameter). In the second case (Macor lens), the lens diameter is only $70 \mathrm{~mm}$ but the high amount of internal reflections strongly degrades the quality of the radiation patterns. Therefore, the lens concept adopted here combines both solutions to take profit from their respective benefits.

\section{A. Lens Design}

The primary source illuminating the dielectric lens is an aperture-coupled microstrip patch antenna printed on HiK 6 substrates $\left(\varepsilon_{\mathrm{r}}=6, h=254 \mu \mathrm{m}\right)$. Its geometry is similar to the one presented in [19] and its dimensions have been determined assuming the patch radiates in the Macor shell at $26 \mathrm{GHz}$ (adopting the same notations as in [19], the dimensions of the patch are the following: $\mathrm{L}_{\text {patch }}=1365 \mu \mathrm{m}, \mathrm{L}_{\text {slot }}=1365 \mu \mathrm{m}$, $\left.\mathrm{W}_{\text {slot }}=315 \mu \mathrm{m}, \mathrm{W}_{\text {line }}=735 \mu \mathrm{m}, \mathrm{L}_{\text {stub }}=1575 \mu \mathrm{m}\right)$.

The theoretical far-field radiation patterns computed with a homemade FDTD solver [20] shows a full width half power beamwidth of about $90^{\circ}$ in both main planes. Therefore, the feed produces a good illumination of the lens surface. These amplitude and phase patterns are used as input parameters for the lens synthesis [12].

The design procedure consists of a global optimization using a genetic algorithm (GA) coupled to a hybrid GO/PO method implemented in 3-D for arbitrarily-shaped multi-shell lenses [12], [15]. We implemented a simple GA based on binary chromosomes, tournament selection and two-point crossover. The antennas generated by GA are evaluated with a cost-function (Fitness function) that is minimized by GA. The Fitness function [see (1)] is calculated by summing the difference between the magnitude of the far-field $\left(E_{G O / P O}\left(\theta_{u}, \varphi_{v}\right)\right)$ calculated with GO/PO for a set of directions of observation $\left(\theta_{u}, \varphi_{v}\right)$, and $\left\{h_{1}\left(\theta_{u}, \varphi_{v}\right), h_{2}\left(\theta_{u}, \varphi_{v}\right)\right\}$ the lower and upper limits of the amplitude template

$$
\begin{aligned}
\text { Fitness }=\sum_{u} \sum_{v} \mid E_{\mathrm{GO} / \mathrm{PO}}\left(\theta_{u}, \varphi_{v}\right) & \\
& -\left.h_{1,2}\left(\theta_{u}, \varphi_{v}\right)\right|^{2} \times \Delta \theta \times \Delta \varphi .
\end{aligned}
$$

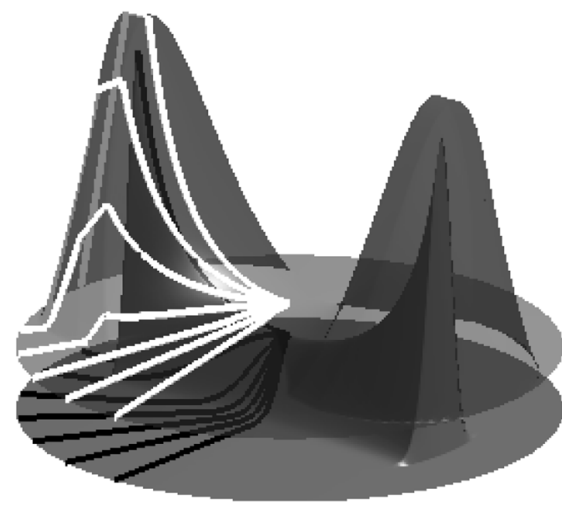

a)

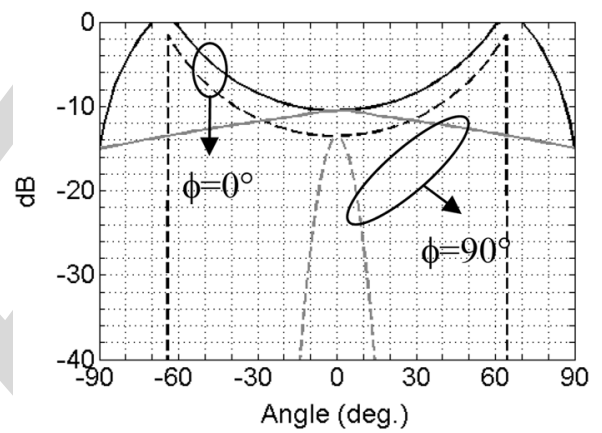

b)

Fig. 8. Power template for the 3-D lens antenna (L2). (a) 3D template. (b) Lower (dashed lines) and upper (solid lines) bounds of the template for two cut-planes $\left(\phi=0^{\circ}\right.$ and $\left.f=90^{\circ}\right)$

The radiation template is represented in Fig. 8 in 3-D and in both principal planes. The Fitness is evaluated from fields computed in seven $\phi$ cut-planes, as highlighted by the black and white lines drawn in Fig. 8(a). Due to the symmetry of the template and feed patterns along $x$ and $y$ axes, the lens shape and patterns are firstly optimized only in one quarter of the radiation space, and then reconstructed in 3-D.

The 3-D optimized shape of the double-shell lens is shown in Fig. 9(a). The corresponding lens profiles computed in four cut planes $\left(\phi=0^{\circ}, 30^{\circ}, 60^{\circ}, 90^{\circ}\right)$ are given in Fig. 9(b). The whole shape is then deduced by symmetry along $x$ and $y$ axes. In the optimization procedure, the thickness of the Rexolite shell is kept constant $(7 \mathrm{~mm})$ and mechanical constraints are taken into account during the synthesis procedure in order to allow the final assembling of both shells.

The GO/PO far-field patterns of the optimized lens (computed without taking into account the influence of internal reflections) are represented in Fig. 10. They are in good agreement with the specifications. Further numerical studies based on FDTD computations have evidenced the presence of acceptable ripples in the lens radiation pattern (Fig. 10) due to the effect of internal reflections in the lens.

The far-field co- and cross-polarization patterns in 3-D computed with GO/PO are given in Fig. 11. The secant-squared pattern is clearly highlighted in Fig. 11(a). The maximum directivity equals $16.4 \mathrm{dBi}$ for $\theta=64^{\circ}$. The maximum level of the cross-polarization component is $-17.7 \mathrm{~dB}$, confirming 


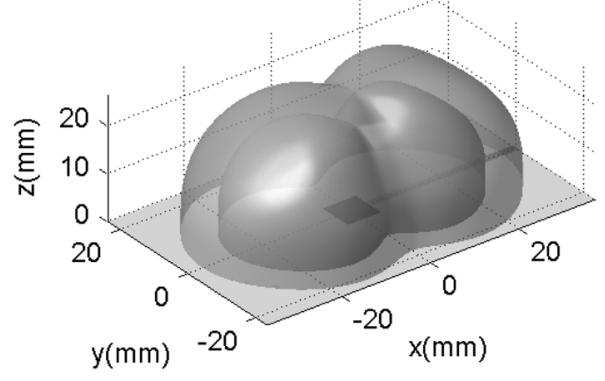

a)

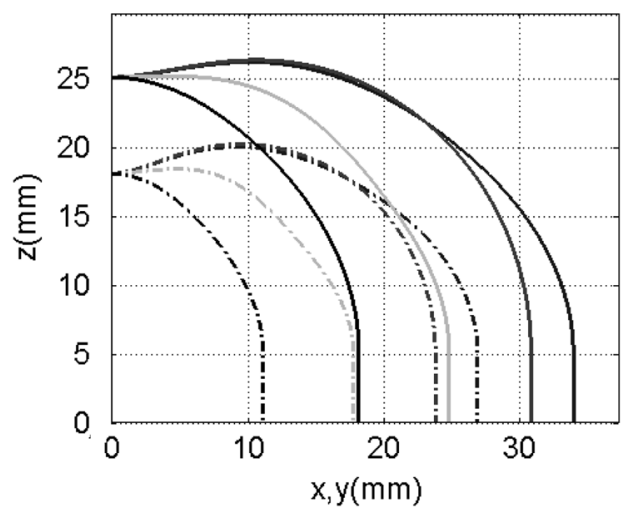

b)

Fig. 9. (a) 3-D view of the optimized L2 lens antenna. (b) Cut-planes ( $\phi=$ $0^{\circ}, 30^{\circ}, 60^{\circ}, 90^{\circ}$ ) of the lens (dotted line: inner interface; solid line: outer interface).
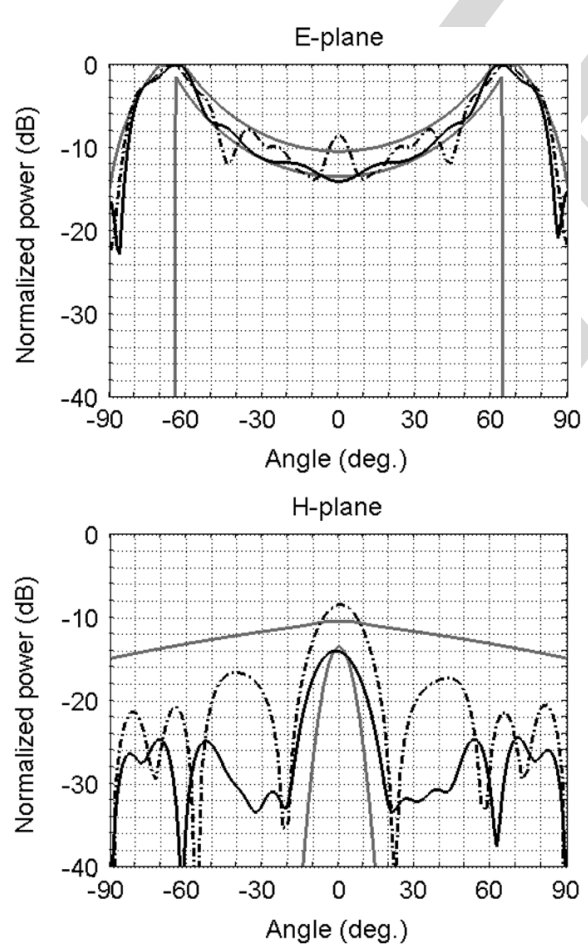

Fig. 10. Co-polarization components computed at $26 \mathrm{GHz}$ in the planes $\phi=$ $0^{\circ}$ (E-plane) and $\phi=90^{\circ}$ (H-plane) with the GO/PO method w/o internal reflections (solid line) and with FDTD (dashed line). The gray lines correspond to the power template specified in Fig. 8.

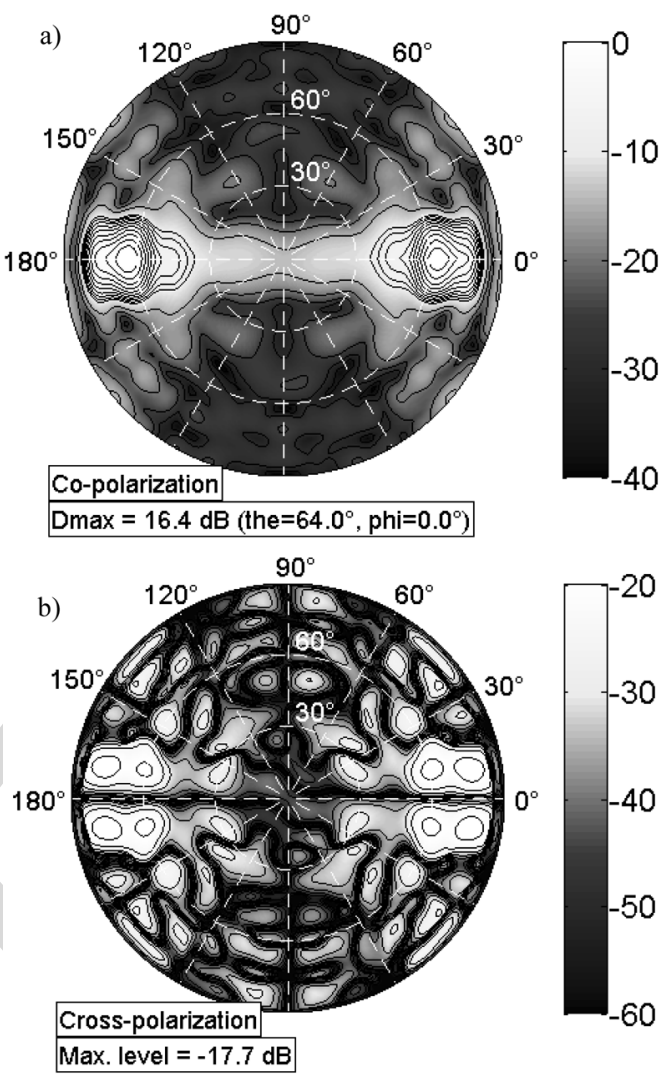

Fig. 11. Radiation patterns (in polar coordinates) of the $\mathrm{L} 2$ lens computed at $26 \mathrm{GHz}$ with the GO/PO method. (a) Co-polarization component; (b) crosspolarization component.

thereby that the double-shell configuration has small depolarization effects, as previously confirmed in Section II. In practice, possible parasitic air gaps could be present between both shells and between the feed and the patch. It is therefore important to anticipate their impact upon the performance of the lens. To this end, we investigated numerically their effects using the FDTD technique, assuming that they have a uniform thickness. The FDTD results have shown that their effects on the radiation performance and reflection coefficient of the lens are negligible provided their thickness is smaller than $300 \mu \mathrm{m}$. However, the presence of an air gap between the patch and the inner shell has a significant impact on its return loss: in that case, the resonant frequency of the antenna is shifted to higher frequencies $(\Delta f=2.5 \mathrm{GHz}$ and $3.1 \mathrm{GHz}$ for air thickness equal to $105 \mu \mathrm{m}$ and $315 \mu \mathrm{m}$, respectively).

\section{B. Measurements}

The antenna prototype has been fabricated with a 3-axis Computer Numerically Controlled (CNC) milling machine [see Fig. 12(a)]. The weight of the lens itself is equal to $80 \mathrm{~g}$ (this value excludes the weight of the feed). The measured input reflection coefficient [Fig. 12(b)] complies with the specifications given in Table I.

Measurements in amplitude at the central frequency $(26 \mathrm{GHz})$ show a good accordance between the theoretical patterns computed with FDTD and experimental data (Fig. 13). The ripples in the co-polarization patterns mainly originate from: (i) the 


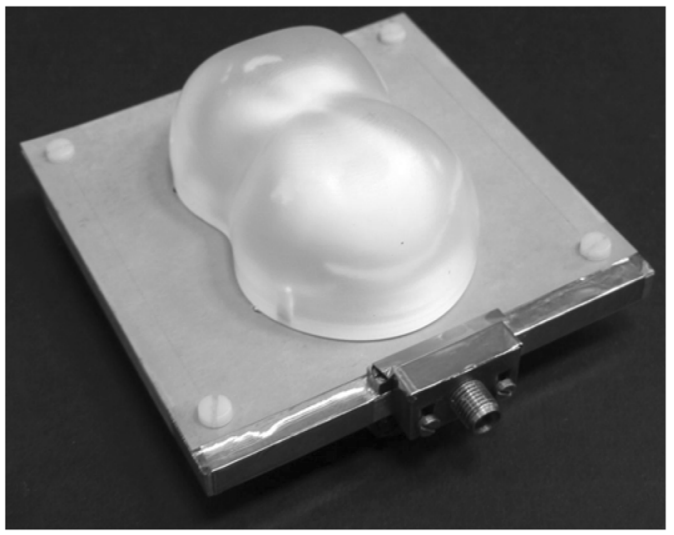

a)

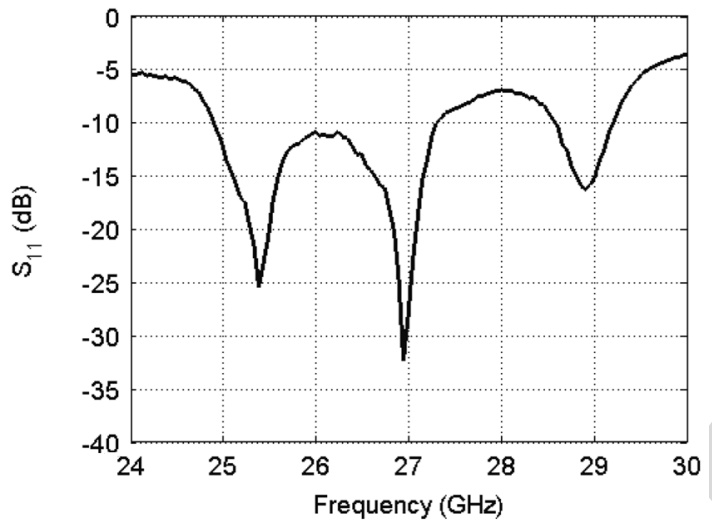

b)

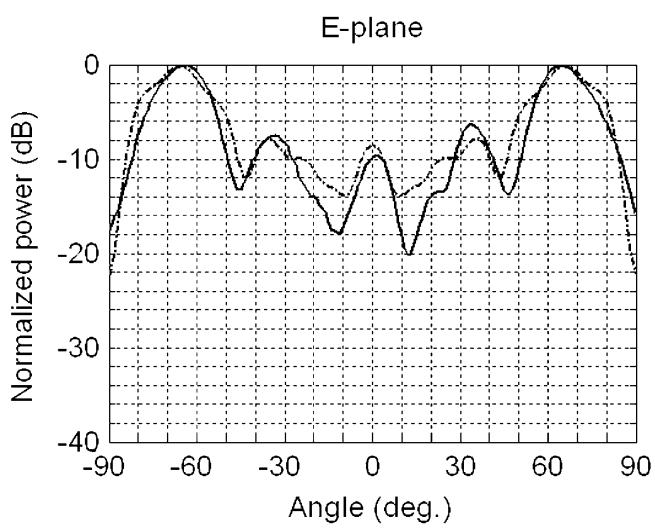

$\mathrm{H}$-plane

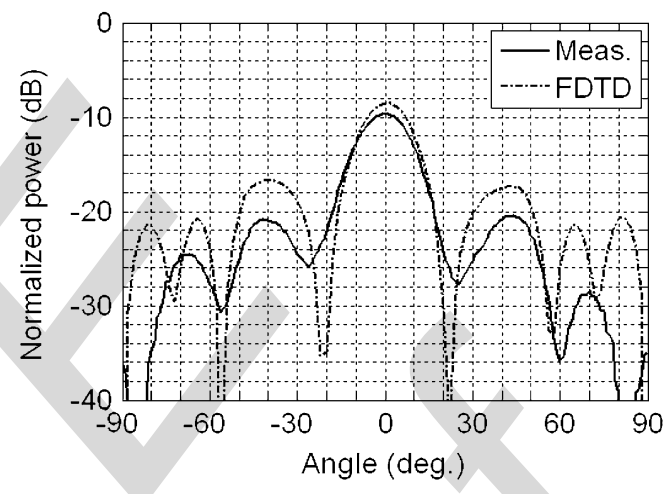

Fig. 13. Measured (solid line) and computed (dashed line) co-polarization component of the $\mathrm{L} 2$ lens antenna at $26 \mathrm{GHz}$.

LEO satellite data link communications at $26 \mathrm{GHz}$. The main design challenge stems from the need to comply simultaneously with the following demanding requirements: 1) dimensions less than $\left.6 \lambda_{0} ; 2\right)$ strongly shaped beam with $\sec (k \theta)$ pattern and minimum depolarization effects; 3 ) enhanced gain; and 4) azimuth scanning requiring no multiple feeds or rotary joints.

Two different lens configurations were proposed. One is based on a new off-centered shaped dome lens and the other one is based on a full 3D double-shell lens. Both solutions comply with specifications, with $11.2 \mathrm{dBi}$ and $12.8 \mathrm{dBi}$ gain, respectively. Gain could be further improved by about $3 \mathrm{~dB}$ using available dielectrics with $10^{-3}$ loss tangent instead of MACOR. This is at least $8 \mathrm{~dB}$ higher than currently used omnidirectional solutions. Lenses are low profile, with less than $103 \mathrm{~g}$ mass, and the configuration is appropriate for mechanical rotation about an appropriate axis for azimuth beam scanning without physical contact of the lens with the fixed feed. This characteristic alone is a marked advantage of the proposed lenses and together with the above referred characteristics makes these lenses unique for the envisaged application.

\section{ACKNOWLEDGMENT}

The authors acknowledge the collaboration from V. Fred, C. Brito, and F. Blanchet for prototype construction, and A. Almeida for prototype measurements. The authors are also grateful to the CNRS/IDRIS,Orsay, France, for the access to their high-performance computing platforms.
In the present work, new lens configurations have been proposed to produce a sector beam with shaping in elevation that enable simple azimuth mechanical scanning, appropriate for 


\section{REFERENCES}

[1] S. Chen, C. du Toit, K. Hersey, D. Pao, V. Karasack, J. Patel, M. Ramesh, C. Sui, and E. Ekelman, "Azimuth scanning Ka-band phased array antenna," in Proc. IEEE AP-S Conf., Boston, MA, 2001, vol. 3, pp. 808-811.

[2] F. Moreira and J. Bergman, "Classical axis-displaced dual-reflector antennas for omnidirectional coverage," IEEE Trans. Antennas Propag., vol. 53, no. 9, pp. 2799-2808, Sep. 2005.

[3] P. Smulders and M. Herben, "A shaped reflector antenna for $60-\mathrm{GHz}$ radio access points," IEEE Trans. Antennas Propag., vol. 49, no. 7, pp. 1013-1015, Jul. 2001.

[4] M. Orefice and P. Pirinoli, "Dual reflector antenna with narrow broadside beam for omnidirectional coverage," Electron. Lett., vol. 29, no. 25, pp. 2158-2159, Dec. 1993.

[5] J. Martínez-Lorenzo, "A shaped and reconfigurable reflector antenna with sectorial beams for LMDS base station," IEEE Trans. Antennas Propag., vol. 54, no. 4, Apr. 2006.

[6] H. Deguchi, H. Kawahara, M. Tsuji, and H. Shigesawa, "Rotational dielectric-dome design for wide-angle scanning applications," in Proc. IEEE AP-S Conf., Washington, DC, 2005, vol. 3A, pp. 638-641.

[7] P. Valentino, C. Rothenberg, and J. Stangel, "Design and fabrication of homogeneous dielectric lenses for dome antenna," in Proc. IEEE AP-S Conf., 1980, vol. 18, pp. 580-583.

[8] C. Abella, J. Peces, M. Marín, J. Martinez, and K. Markus, "Development of a compact antenna for global earth coverage," in Proc. 23rd Eur. Microwave Conf., Madrid, Spain, Oct. 1993, pp. 906-908.

[9] D. Lemaire, C. A. Fernandes, P. Sobieski, and A. Barbosa, "A method to overcome the limitations of GO in the design of axis-symmetrical lenses," Int. J. Infrared Millimetre Waves, vol. 17, no. 8, pp. 1377-1390, Aug. 1996.

[10] L. Martin, A. Pierre, L. Albo, G. Caille, G. Piton, and J.-M. Lopez, "Matériaux diélectriques hautes performances pour antennes spatiales á lentille," presented at the 7émes Journées de Caractérisation Micro-Ondes et Matériaux, JCMM'2002, Toulouse, France, Mar. 20-21, 2002.

[11] B. Barés and R. Sauleau, "Electrically-small shaped integrated lens antennas: A study of feasibility in Q-band," IEEE Trans. Antennas Propag., vol. 55, no. 4, pp. 1038-1044, Apr. 2007.

[12] G. Godi, R. Sauleau, L. Le Coq, and D. Thouroude, "Design and optimization of three dimensional integrated lens antennas with genetic algorithm," IEEE Trans. Antennas Propag., vol. 55, no. 3, pp. 770-774, Mar. 2007, part 1.

[13] C. A. Fernandes, , L. Godara, Ed., Shaped-Beam Antennas. Boca Raton, FL: CRC Press, 2002, Ch. 15 in Handbook of Antennas in Wireless Communications.

[14] M. Silveirinha and C. A. Fernandes, "Shaped double-shell dielectric lenses for wireless millimetrewave communications," in Proc. IEEE AP-S Conf., Salt Lake City, UT, 2000, vol. 3, pp. 1674-1677.

[15] G. Godi, R. Sauleau, and L. Le Coq, "A shaping technique of substrate lens antennas with genetic algorithm,", presented at the 11th Int. Symp. on Antenna Technology and Applied Electromagnetics, ANTEM'2005, Saint-Malo, France, Jun. 15-17, 2005.

[16] J. Costa, C. Fernandes, G. Godi, R. Sauleau, and H. Legay, "Benchmark of lens antennas for Ka-band global Earth observation from LEO satellites," presented at the European Conf. on Antennas and Propagation-EUCAP 2006, Nice, France, Nov. 2006.

[17] C. A. Fernandes, "Design of shaped lenses for non-symmetric cells in MBS," in Proc. IEEE AP-S Conf., Orlando, FL, 1999, vol. 3, pp. 2440-2443.

[18] J. Costa, M. Silveirinha, and C. A. Fernandes, "Design and analysis of double-shell axial-symmetric integrated lens antennas for space applications," in Proc. 28th ESA Antenna Workshop on Space Antenna Systems and Technologies, Noordwijk, The Netherlands, May 2005, vol. 2, pp. 865-872.

[19] G. Godi, R. Sauleau, and D. Thouroude, "Performance of reduced size substrate lens antennas for millimetre-wave communications," in IEEE Trans. Antennas Propag., Apr. 2005, vol. 53, no. 4, pp. 1278-1286.

[20] R. Sauleau, C. Guiffaut, P. Coquet, K. Mahdjoubi, and J.-P. Daniel, "FDTD modeling on high performance vector/parallel computers. Application to the analysis of millimeter wave printed antennas and focusing devices," in Proc. 8th Int. Conf. on Advances in Communications and Control, COMCON8, Telecommunications/Signal Processing, Crete, Greece, June 25-29, 2001, pp. 329-347.
[21] A. V. Boriskin, G. Godi, R. Sauleau, and A. I. Nosich, "Small hemielliptic dielectric lens antenna analysis in 2-D: Boundary integral equations vs. geometrical and physical optics," IEEE Trans. Antennas Propag., to be published.

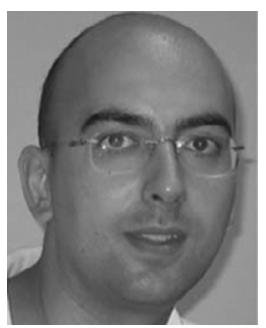

Jorge R. Costa ( ' $\left.^{\prime} 97-M^{\prime} 03\right)$ was born in Lisbon, Portugal, in 1974. He received the Licenciado and $\mathrm{Ph} . \mathrm{D}$. degrees in electrical engineering from the Instituto Superior Técnico, Lisbon, Portugal, in 1997 and 2002, respectively

$\mathrm{He}$ is currently a Researcher at the Instituto de Telecomunicações, Lisbon, Portugal. He is also an Assistant Professor at the Departamento de Ciências e Tecnologias da Informação, Instituto Superior das Ciências do Trabalho. His present research interests switchs and RFID antennas. include lenses, reconfigurable antennas, MEMS

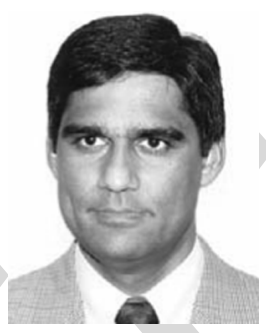

Carlos A. Fernandes (S'86-M'89) received the Licenciado, M.Sc., and Ph.D. degrees in electrical and computer engineering from the Instituto Superior Técnico (IST), Technical University of Lisbon, Lisbon, Portugal, in 1980, 1985, and 1990, respectively.

In 1980, he joined the Department of Electrical and Computer Engi $\equiv \mathrm{g}$, IST, where he is presently a Full Professor Inzute areas of microwaves, radio wave propagation and antennas. He is also a Senior Researcher at the Instituto de Telecomunicações, where he is the Coordinator of the Wireless Communications scientific area. He has been the leader of antenna activity in National and European Projects such as RACE 2067-MBS (Mobile Broadband System), ACTS AC230-SAMBA (System for Advanced Mobile Broadband Applications) and ESA/ESTEC-ILASH (Integrated Lens Antenna Shaping). He has coauthored a book, a book chapter, and several technical papers in international journals and conference proceedings, in the areas of antennas and radiowave propagation modeling. His current research interests include artificial dielectrics, dielectric antennas for millimeter wave applications, and propagation modeling for mobile communication systems.

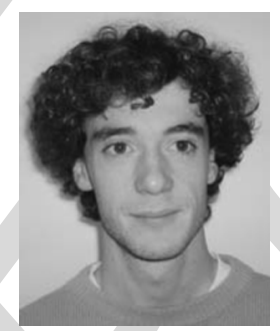

Gaël Godi was born in France in 1980. He received the electronics engineering degree and the M.S. degree in electronics from the National Institute of Applied Science (INSA) of Rennes, France, in 2003 and the Ph.D. degree from the University of Rennes 1 , France, in 2006.

His research interests include the analysis and optimization of dielectric lens antennas for millimeter-wave applications.AUTHOR: WHAT ORGANIZATION IS GODI WITH NOW?

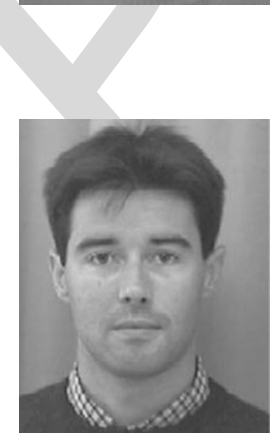

Ronan Sauleau (M'04-SM'06) received the Electronic Engineering and Radiocommunications Degree and the French DEA degree in electronics from the Institut National des Sciences Appliquées (INSA), Rennes, France, in 1995, the Aggregation degree from Ecole Normale Supérieure de Cachan, France, in 1996, and the Doctoral degree in signal processing and telecommunications from the IETR, University of Rennes 1, Rennes, in 1999.

Between September 2000 and 2005, he was an Assistant Professor at the University of Rennes 1, where, since November 2005, he has been an Associate Professor. His main current fields of interest of are numerical modeling, millimeter-wave printed and reconfigurable (MEMS) antennas, lens-based focusing devices, periodic structures (electromagnetic bandgap materials and metamaterials) and biological effects of millimeter waves. He has received three patents and is the author or coauthor of 42 journal papers and more than 110 contributions to national and international conferences and workshops. 
Dr. Sauleau received the 2004 ISAP Conference Young Researcher Scientist Fellowship (Japan) and the first Young Researcher Prize in Brittany, France, in 2001 for his research work on gain-enhanced Fabry-Perot antennas. In September 2007, he was elevated to Junior Member of the "Institut Universitaire de France.'

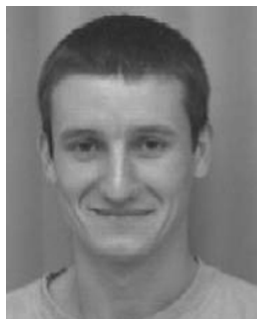

Laurent Le Coq received the Electronic Engineering and Radiocommunications degree and the French DEA degree (M.Sc.) in electronics and the Ph.D. from the National Institute of Applied Science (INSA), Rennes, France, in 1995 and 1999, respectively.

In 1999, he joined the Institute of Electronics and Telecommunications of Rennes (IETR), University of Rennes 1, as a Research Lab Engineer, where he is responsible for measurement technical facilities up to $110 \mathrm{GHz}$

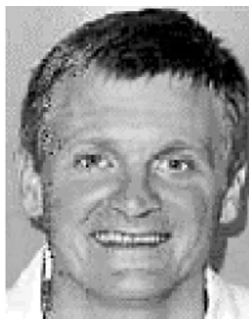

Hervé Legay was born in 1965. He received the Electrical Engineering Degree and the Ph.D. degree from the National Institute of Applied Sciences (INSA), Rennes, France, in 1988 and 1991, respectively.

For two years, he was a Postdoctoral Fellow at the University of Manitoba, Winnipeg, Canada, where he developed innovating planar antennas. He joined Alcatel Space, Toulouse, France, in 1994, which is now Thales Alenia Space. He initially conducted studies in the areas of military telecommunication advanced antennas and antenna processing. He currently leads research projects in integrated front ends and reflectarray antennas and coordinates the collaborations with academic and research partners in the area of antennas. He was member of the Alcatel Technical Academy. 